\title{
Social comparison and envy on social media: A critical review
}

\author{
Adrian Meier \\ School of Business, Economics and Society, FAU Erlangen-Nuremberg, Germany \\ Benjamin K. Johnson \\ Department of Advertising, University of Florida, USA
}

This is a post-print version of the peer-reviewed manuscript as published in Current Opinion in Psychology. Please find the published version here:

https://doi.org/10.1016/j.copsyc.2022.101302

\section{Corresponding author:}

Adrian Meier, adrian.meier@,fau.de

\section{ORCIDs:}

Adrian Meier: https://orcid.org/0000-0002-8191-2962

Benjamin K. Johnson: https://orcid.org/0000-0003-0588-221X

\section{Conflict of interest:}

This research did not receive any funding. The authors are not aware of any conflict of interest.

\section{CRediT author contribution statement:}

Adrian Meier: Conceptualization; literature search; writing and revising the original draft Benjamin Johnson: Conceptualization; literature search; reviewing, editing, and commenting draft 


\section{Highlights}

- Social media (SM) supposedly make social comparison, envy, and well-being worse

- This review finds mixed and inconsistent evidence for this claim

- Earlier studies show comparisons and envy are common on SM and linked to ill-being

- Recent studies find positive, person-specific, conditional, and reciprocal effects

- Specific causes, online/offline differences, and user agency remain open questions 


\begin{abstract}
There is both public and scholarly concern that (passive) social media use decreases well-being by providing a fertile ground for harmful (upward) social comparison and envy. The present review critically summarizes evidence on this assumption. We first comprehensively synthesize existing evidence, including both prior reviews and the most recent publications (2019 to 2021). Results show that earlier research finds social comparison and envy to be common on social media and linked to lower well-being. Yet, increasingly, newer studies contradict this conclusion, finding positive links to well-being as well as heterogeneous, person-specific, conditional, and reverse or reciprocal effects. The review identifies four critical conceptual and methodological limitations of existing evidence, which offer new impulses for future research.
\end{abstract}

Keywords: social comparison, envy, well-being, social media, passive use, critical review 


\section{Introduction}

Social media (SM) such as Facebook, Instagram, and TikTok continue to attract billions worldwide while concerns about their impact on users' well-being are unabated [1]. Recent overviews $[2,3,4]$ of meta-analyses on this issue find overall small negative associations between social media use (SMU) and some, but not all, well-being indicators among adolescents [4] and young adults [2, 3]. Yet associations are heterogeneous and likely depend on numerous boundary conditions and mechanisms $[3,4]$. Several reviews $[5,6,7]$, plus Facebook's own research $[8,9]$, point to social comparison and envy as key mechanisms.

Social comparison is "the process of thinking about information about one or more other people in relation to the self' [10]. Comparisons represent a fundamental human cognition [11] occurring across cultures [12], helping humans navigate social groups by providing them with information about their own relative standing [13]. Despite these benefits, upward comparisons - with those who do better than oneself — have been identified as potentially problematic [14], especially on SM [7]. As users present a positively-biased version of themselves online [15], upward comparisons are assumed to be more frequent and harmful on SM [7]. They may particularly elicit envy, "the pain caused by the good fortune of others," which can impair well-being $[16,17]$.

Whereas this negative view dominated research on SM upward comparison and envy in the 2010 s, recent studies also point to positive outcomes such as inspiration $[18,19,20]$. More generally, authors $[18,20,21,22,23]$ —including original proponents $[6,24]$ — have begun to question whether the effects of passive SMU (i.e., just browsing SM without interacting) are as detrimental as previously assumed [cf. 7] and whether "active" versus "passive" use is a useful dichotomy to explain well-being effects. 
Our review seeks to advance this debate, first, with a synthesis of prior reviews and recent key studies, focused on 2019-2021. The goal is to provide both a comprehensive update and critical appraisal of the most relevant evidence. Note that we exclude studies solely investigating appearance comparison or body image because an up-to-date review of this literature appears in this special issue (see Vandenbosch et al.). Instead, our review includes studies measuring all non-specific social comparisons and envy, which may be elicited by various self-presentations of people's work, leisure, travel, health, fitness, or appearance, among others. Second, and building on this evidence synthesis, we identify and critically discuss four major limitations of the literature, which point to crucial questions for future research.

\section{State of the evidence}

\subsection{Reviews and meta-analyses}

Research syntheses on SM social comparisons, envy, and well-being present a complex set of conclusions $[5,6,7,17,25,26,27]$. Current consensus is that social comparison and envy are frequent experiences for SM users, and both are associated with lower well-being, albeit depending on measurement. In a meta-analysis of $k=22$ effects from 13 publications $(N=$ 11,199) [26], Facebook social comparisons and well-being (e.g., affect, self-esteem, life satisfaction) showed a small to moderate negative association $(r=-.20,95 \%$ CI [-.29, -.11]). In a second meta-analysis of Facebook social comparisons and depressive symptoms [27], general social comparisons showed a small to moderate positive association $(r=.23,95 \% \mathrm{CI}[.12, .34], k$ $=8$ effects from 5 publications, $N=1,715$ ), and upward comparisons showed a moderate positive association with depressive symptoms $(r=.33,95 \%$ CI $[.20, .47], k=6$ effects from 5 publications, $N=2,298)$. 
Reviews disagree, however, about severity and implications. While most interpret the associations to suggest detrimental effects of SM on well-being transmitted by social comparison and envy $(6,7,25,26,27)$, others are more cautious, emphasizing complexity and limitations in current findings $(5,17)$. In the following, we complement this overview with key findings from recent studies, most not included in prior reviews.

\subsection{Positive correlates of social comparison and envy}

In contrast to earlier SM research that assumed social comparison and envy to be entirely negative experiences [7], multiple recent studies suggest they can also be positive [18, 20,28]. This is explained through comparisons focusing either on assimilation (similarities with the target) or contrast (differences with the target). Whereas upward assimilation and downward contrast are expected to improve well-being, upward contrast and downward assimilation are expected to decrease it [14]. Similarly, envy is sometimes distinguished into a more assimilative, beneficial emotion (benign envy) and a more contrastive, harmful one (malicious envy) [16]. Most research so far assumes that SM are particularly harmful by triggering upward contrast rather than assimilation, and malicious rather than benign envy [7, 17]. Upward contrast has also been meta-analytically identified as humans' default social comparison reaction-at least in experimental paradigms [14]. Whether this laboratory finding generalizes to peoples' face-toface or SM comparisons in daily life remains largely unclear.

Intriguingly, recent evidence suggests assimilative experiences are quite common for SM users: For instance, two experiments [18] and three surveys [29, 30, 31] observe benign envy to be more common than malicious envy on SM. They also find inspiration-a positive motivational outcome of assimilative upward comparison — to be frequently associated with SMU. Another survey corroborates upward comparison on Facebook as more strongly associated 
with assimilative than contrastive emotions [28]. In a three-wave panel survey among adolescents, higher self-reported exposure to positively-biased SM content was associated with increased inspiration four months later, but not increased envy [15]. However, higher SM social comparison was associated with higher envy four months later, suggesting complex effects. The positive side of SM comparisons was also apparent during the COVID-19 pandemic: in a three-

wave panel survey during lockdown in Italy, online social comparison predicted improvement in anxiety, stress, loneliness, and life satisfaction levels over time, likely because SM granted opportunities for lateral or downward comparisons, seeing that others were suffering similarly or worse from lockdowns [32].

Overall, and consistent with some earlier research [19,33], recent studies indicate that positive reactions to passive SMU are plausible and not uncommon. Yet most of these studies relied on small cross-sectional surveys and the occasional experiment. In the following, we thus review key studies employing more advanced methods and larger samples.

\subsection{Heterogeneous, person-specific, and conditional effects}

Recent publications used idiographic $N=1$ methods to study passive SMU and wellbeing [34, 35], or envy and inspiration [20]. These studies offer new insights into person-specific effects of SMU on well-being, that is, for how many users the effects of SMU are negative, positive, or null. Experience sampling methods (ESM) find that only $10-20 \%$ of Dutch adolescents felt worse after passive SMU, with the rest either feeling better or the same [34]. Malicious envy and inspiration each occurred in a third of passive SMU episodes, suggesting similar prevalence [20].

A prior review concluded that "positive consequences may well be the exception rather than the rule" on SM [7]. Together, the person-specific evidence and the positive correlates 
described above challenge this widely held view of "negative social comparison" and malicious envy as the predominant reactions on SM. Rather, effects are often conditioned on the SM used $[34,36$, but see 37], SM content [8, 18, 38, 39], gender and age [8, 40], and culture [8, 12$]$.

\subsection{Longitudinal evidence}

In addition to the person-specific studies [20, 34, 35], several new publications have investigated the effects of SMU on social comparison, envy, and well-being using longitudinal methods, such as panel surveys or ESM. Earlier studies $[4,6,7]$ found negative longitudinal associations of (passive) SMU with well-being, and that upward comparison was associated negatively with well-being and positively with envy [see also $36,37,41]$.

In contrast, several recent studies point to reverse or reciprocal effects between SMU, social comparison, envy, and well-being [15, 40, 42, 43, 44]. For instance, an ESM study [42] observed that while passive SMU and depressive symptoms were correlated within-episode (i.e., a two-hour window), depressive symptoms predicted passive SMU over time but not vice versa. A two-wave survey found depressive symptoms to predict passive Facebook use and envy one year later, but no reciprocity [43]. Another found time on Facebook increased depressive symptoms a year later, which increased time on Facebook another year later [44]. A three-wave panel survey further found that SM social comparison and inspiration predicted exposure to attractive appearances on SM four months later, with minimal reverse effects of exposure [15]. Other panel and ESM studies, however, mostly observe no reverse or reciprocal effects [32, 36, 37, 45]. Overall, the longitudinal evidence on (passive) SMU, social comparison, envy, and wellbeing is conflicting. It suggests a complex interplay between user experiences across short and long timeframes.

\subsection{International evidence from industry research}


Beyond independent academic research, SM companies themselves increasingly investigate SM social comparison and well-being. A recent study combined survey responses with trace data from nearly 38,000 Facebook users across 18 countries [8]. Results indicate that social comparison is an occasional experience on Facebook. It is more frequent among users who are younger, have more friends, spend more time on the application and especially user profiles, and those who encounter more social, highly-liked, and positive content in their feeds [8].

However, country was the strongest predictor of comparison frequency on Facebook, with a more pronounced effect than all other factors combined. Facebook comparisons were more frequent in Eastern (more collectivist) than Western (more individualist) countries [8]. Country also interacted with gender: men in most Eastern (e.g., India) and women in several Western (e.g., UK, USA) countries reported more frequent comparison, suggesting a crucial role of both culture and gender [12]. Additionally, almost a quarter of all participants viewed a post that made them feel worse in comparison within the last two weeks, and they reported a mix of affective reactions [8].

Internal research by Meta Platforms, leaked to the public in 2021, provides additional insights into Instagram use [1,9]. Multiple studies, including an international cross-sectional survey $(N>22,000)$ and in-depth interviews with adolescents, showed that teens perceived social comparison via Instagram as a key issue for their well-being. However, they also perceived their Instagram use to be a net benefit. These findings overall underline the importance but also the heterogeneous and conditional nature of SM social comparison effects. Although industry research should be interpreted with caution, the findings converge with independent academic studies.

\section{Limitations and open questions}


The evidence reviewed above has crucial limitations which inform our overarching questions to future research: (a) What are the differences between online and offline social comparison and envy? (b) What, exactly, causes social comparison and envy on SM, and their well-being effects? (c) How much does user agency matter? (d) What methodological issues need overcome?

\subsection{Online and offline comparisons}

Although extensive research shows that comparisons and envy occur on SM, there is insufficient demonstration that SM change anything about them. First, most studies simply replicate comparison and envy reactions on SM where predictors and outcomes "mirror findings from the offline context" [17], which does not show how SM are supposedly more harmful [7]. Indeed, while a positivity bias is well-documented for SM self-presentations [15], so it is for human communication in general [46]. Second, the claim that "social comparisons take place at an unprecedented rate and scale" due to SM [7] lacks evidence. Initial studies show online social comparison produces more short-term negative affect, yet offline comparison may be more prevalent and consequential for well-being $[37,47]$. That SM have changed the frequency, intensity, direction, or foci of social comparison and envy_compared to general human experience - is plausible but remains largely untested. Future studies need to include nonmediated benchmarks $[36,47]$ and isolate SM's unique characteristics to make this case.

\subsection{Causes of social comparison and envy}

Existing research remains vague on what exactly about SM triggers social comparison and envy, or what supposedly causes them to be more frequent, intense, or harmful. Through the lens of computer-mediated communication [2], social comparison and envy could be shaped by ubiquitous mobile access [36]; the sheer amount of SMU across applications [35, 37]; 
characteristics and culture of individual apps [9,30]; design features signaling social status (e.g., likes) [48]; whether one interacts with strangers, influencers, or likeminded friends [8, 28, 31, 33, 41, 49]; characteristics of SM messages, such as content (e.g., fitspiration vs. travel) or modality (e.g., visual vs. textual), or their algorithmic ranking $[17,18,22,38,39] —$ or any combination of these factors.

Given this complexity of potential causes, a simple dichotomization into "active" and "passive" SMU seems obsolete. Research increasingly suggests that effects of passive and active SMU are undifferentiated $[23,35]$. Authors further challenge the utility of the active/passive dichotomy on conceptual and operational grounds $[6,22,23,24,50]$. In addition, and as noted by others [6,7], SMU may be linked to well-being via alternative mechanisms, particularly emotional contagion [51]. Overall, future research will need to pinpoint potentially multifaceted causes for SM comparisons and envy, and their unique effects on well-being.

\subsection{User agency}

Almost all prior research has ignored the role of users' agency, that is, the various forms of choice and control individuals are able to exert over their SM experiences. Instead, studies (implicitly) follow a dose-response model where SM users are mere recipients of content. Yet, experiments and surveys from the selective exposure paradigm indicate that SM comparisons are driven by motivations and moods $[52,53]$. Users are, for instance, motivated to obtain positive outcomes by gravitating towards inspiring content or (un)following accounts $[8,15,30,53]$. Eye-tracking research indicates users pay selective attention during passive SMU, hence partially modulating their exposure $[21,22]$. However, agency on SM is clearly constrained by ranking algorithms and others' posts and feedback $[8,13]$. Agency is also no panacea against inadvertent negative effects of SMU [7, 54]. Still, identifying design interventions that increase users' 
control over exposure, comparison and envy reactions, or the outcomes of unintentional comparisons, will enhance both our knowledge of these processes and users' experiences [48, 49].

\subsection{Methodological issues}

Beyond these largely conceptual challenges, we face methodological ones $[17,52,55]$. Self-report measures of SM social comparison often fail to specify comparison direction (upward vs. downward) and focus (assimilation vs. contrast), despite their differential outcomes [14]. Various studies conflate upward comparison with negative outcomes, inflating negative associations with well-being [52]. To avoid such confirmation bias in research design, measures must instead clearly separate SM comparison direction from comparison focus and outcomes $[18,55]$. Furthermore, studies often adapt social comparison orientation scales - measures of immutable traits - to SM contexts. These are then improperly positioned as mediators or outcomes [55], when state measures would be necessary instead [18]. Similarly, envy scales differ in whether they include a social comparison component or focus on affective or motivational aspects of the emotion [17]. This incoherent measurement reflects psychology's dissensus on the nature of envy [16]. Beyond choosing or developing less biased self-report scales, future SM research should also use behavioral measures of SMU, selective exposure, and selective attention $[8,22,52]$.

\section{Conclusion}

Do the highlight reels on social media make us feel worse due to upward comparison and envy? This question has received considerable attention and is regarded crucial by SM companies, yet this evidence review provides a mixed answer. Earlier studies and initial small meta-analyses confirmed that social comparison and envy were common on SM and linked to 
lower well-being. Yet, recent studies increasingly contest this position and point to effects of (passive) SMU, social comparison, or envy on well-being as (a) positive; (b) heterogeneous and person-specific; (c) reverse or reciprocal; and (d) conditioned, e.g., on culture, gender, and SM content. In addition, studies leave unanswered (e) whether these effects are different on SM or only replicate human psychology in a technological context; (f) what exactly about SM causes effects; (g) how users' agency modulates effects; and (h) whether effects replicate when less biased measurements are used. Given contradictory findings and limitations, we cannot conclude that SM are indeed "worse"-we simply do not know yet. In any case, recommending users to just "stop comparing" on SM seems futile, given how deeply this experience is part of the human condition. Instead, efforts of researchers, companies, and users alike can be directed towards identifying ways in which SM design and usage practices can foster positive, inspiring comparisons and reduce negative ones $[8,49,56,57]$. 


\section{References and recommended reading}

Papers of particular interest, published within the period of review, are highlighted as:

* of special interest

** of outstanding interest

[1] G. Wells, J. Horwitz, D. Seetharaman, Facebook knows Instagram is toxic for teen girls, company documents show, 2021. https://www.wsj.com/articles/facebook-knows-instagramis-toxic-for-teen-girls-company-documents-show-11631620739.

[2] A. Meier, L. Reinecke, Computer-mediated communication, social media, and mental health: A conceptual and empirical meta-review, Communication Research 48 (2021) 11821209. https://doi.org/10.1177/0093650220958224.

[3] P.M. Valkenburg, Social media use and well-being: What we know and what we need to know, Current Opinion in Psychology (2021). https://doi.org/10.1016/j.copsyc.2021.12.006.

[4] P.M. Valkenburg, A. Meier, I. Beyens, Social media use and its impact on adolescent mental health: An umbrella review of the evidence, Current Opinion in Psychology 44 (2022) 58-68. https://doi.org/10.1016/j.copsyc.2021.08.017.

[5] J.B. Bayer, P. Triệu, N.B. Ellison, Social media elements, ecologies and effects, Annu. Rev. Psychol. 71 (2020) 10.1-10.27. https://doi.org/10.1146/annurev-psych-010419-050944.

[6] E. Kross, P. Verduyn, G. Sheppes, C.K. Costello, J. Jonides, O. Ybarra, Social media and well-being: Pitfalls, progress, and next steps, Trends in Cognitive Sciences (2020). https://doi.org/10.1016/j.tics.2020.10.005.

*[7] P. Verduyn, N. Gugushvili, K. Massar, K. Täht, E. Kross, Social comparison on social networking sites, Current Opinion in Psychology 36 (2020) 32-37. https://doi.org/10.1016/j.copsyc.2020.04.002. 
This review presents the state of research on SM social comparison up until 2020. It can be read side by side with our review to get a full picture of the current evidence base and (dis)agreements in this field.

[8] M. Burke, J. Cheng, B. de Gant, Social comparison and Facebook: Feedback, positivity, and opportunities for comparison, in: Proceedings of the $2020 \mathrm{CHI}$ conference on human factors in computing systems, Honolulu HI USA, ACM, New York, NY, 2020, Article 355.

[9] Facebook, What our research really says about teen well-being and Instagram, 2021.

[10] J.V. Wood, What is social comparison and how should we study it?, Pers Soc Psychol Bull 22 (1996) 520-537. https://doi.org/10.1177/0146167296225009.

[11] M. Baldwin, T. Mussweiler, The culture of social comparison, Proc. Natl. Acad. Sci. U. S. A. 115 (2018) E9067-E9074. https://doi.org/10.1073/pnas.1721555115.

**[12] J. Cheng, M. Burke, B. de Gant, 2021. Country differences in social comparison on social media. Proc. ACM Hum.-Comput. Interact. 4, 270. https://doi.org/10.1145/3434179.

Large-scale, international survey coupled with trace data and interviews from Facebook users. Country was by far the strongest demographic predictor of comparison frequency. Women in Western countries, and men in Eastern countries, reported more social comparison on Facebook. The relationship between like counts and comparison frequency depended on country.

[13] C.R. Blease, Too many 'friends,' too few 'likes'? Evolutionary psychology and 'Facebook depression', Review of General Psychology 19 (2015) 1-13. https://doi.org/10.1037/gpr0000030.

[14] J.P. Gerber, L. Wheeler, J. Suls, A social comparison theory meta-analysis $60+$ years on, Psychological Bulletin 144 (2018) 177-197. https://doi.org/10.1037/bul0000127. 
[15] L. Schreurs, A. Meier, L. Vandenbosch, Exposure to the positivity bias and adolescents' differential longitudinal links with inspiration and envy depending on social media literacy (2021). https://doi.org/10.31219/osf.io/am2dj.

[16] J. Crusius, M.F. Gonzalez, J. Lange, Y. Cohen-Charash, Envy: An adversarial review and comparison of two competing views, Emotion Review 12 (2020) 3-21. https://oi.org/10.1177/1754073919873131.

*[17] H. Wenninger, C.M. Cheung, M. Chmielinski, 2021. Understanding envy and users' responses to envy in the context of social networking sites: A literature review. International Journal of Information Management 58, 102303. https://doi.org/10.1016/j.ijinfomgt.2020.102303. An up-to-date, interdisciplinary, and nuanced review on the role of envy in SM environments. It concludes there is no evidence indicating envy is considerably different on SM than outside of this technological context. The review also looks beyond well-being outcomes, discussing, for instance, the importance of SM envy for advertising effects (e.g., purchase intentions).

**[18] A. Meier, A. Gilbert, S. Börner, D. Possler, Instagram inspiration: How upward comparison on social network sites can contribute to well-being, J Communication 70 (2020) 721-743. https://doi.org/10.1093/joc/jqaa025.

Two experiments test whether viewing more vs. less visually optimized nature/travel Instagram posts triggers upward comparison, envy, and inspiration. Results overall suggest that more optimized pictures elicit stronger upward comparison, benign and malicious envy, but also inspiration. Upward comparison and benign (but not malicious) envy were linked to higher inspiration, which was accompanied by increased well-being. 
[19] S.Y. Park, Y.M. Baek, Two faces of social comparison on Facebook: The interplay between social comparison orientation, emotions, and psychological well-being, Computers in Human Behavior 79 (2018) 83-93. https://doi.org/10.1016/j.chb.2017.10.028.

**[20] P.M. Valkenburg, I. Beyens, J.L. Pouwels, I.I. van Driel, L. Keijsers, Social media browsing and adolescent well-being: Challenging the "passive social media use hypothesis", Journal of Computer-Mediated Communication 27 (2021). https://doi.org/10.31234/osf.io/gzu3y.

Among a large sample of Dutch adolescents, this ESM study finds considerable heterogeneity in the within-person associations between passive SMU and envy, inspiration, and enjoyment. Idiographic $N=1$ analyses confirmed the common hypothesis, "passive SMU leads to lower wellbeing," for only $20 \%$ of adolescents, hence challenging the generalizability of this assumption.

[21] N.B. Ellison, P. Triệu, S. Schoenebeck, R. Brewer, A. Israni, Why we don’t click: Interrogating the relationship between viewing and clicking in social media contexts by exploring the "non-click", J Comput-Mediat Comm 25 (2020) 402-426. https://doi.org/10.1093/jemc/zmaa013.

[22] P. Triệu, N.B. Ellison, S.Y. Schoenebeck, R.N. Brewer, Implications of Facebook engagement types and feed's social content for self-esteem via social comparison processes, Social Media + Society 7 (2021) 205630512110424. https://doi.org/10.1177/20563051211042400.

[23] P.M. Valkenburg, I.I. van Driel, I. Beyens, The associations of active and passive social media use with well-being: A critical scoping review, New Media \& Society (2021). https://doi.org/10.1177/14614448211065425. 
[24] P. Verduyn, N. Gugushvili, E. Kross, Do social networking sites influence well-being? The extended active-passive model, Curr Dir Psychol Sci (2021). https://doi.org/10.1177/09637214211053637.

[25] H.-V. Krause, K. Baum, A. Baumann, H. Krasnova, Unifying the detrimental and beneficial effects of social network site use on self-esteem: a systematic literature review, Media Psychology 24 (2021) 10-47. https://doi.org/10.1080/15213269.2019.1656646.

[26] F.-R. Yang, C.-F. Wei, J.-H. Tang, Effect of Facebook social comparison on well-being: A meta-analysis, Journal of Internet Technology 20 (2019) 1829-1836. https://doi.org/10.3966/160792642019102006013.

[27] S. Yoon, M. Kleinman, J. Mertz, M. Brannick, Is social network site usage related to depression? A meta-analysis of Facebook-depression relations, J. Affect. Disord. 248 (2019) 65-72. https://doi.org/10.1016/j.jad.2019.01.026.

[28] L.P. Tosun, E. Kaşdarma, Passive facebook use and depression: A study of the roles of upward comparisons, emotions, and friendship type, Journal of Media Psychology 32 (2020) 165-175. https://doi.org/10.1027/1864-1105/a000269.

[29] K. Latif, Q. Weng, A.H. Pitafi, A. Ali, A.W. Siddiqui, M.Y. Malik, Z. Latif, 2021. Social comparison as a double-edged sword on social media: The role of envy type and online social identity. Telematics and Informatics 56, 101470. https://doi.org/10.1016/j.tele.2020.101470.

[30] A. Meier, S. Schäfer, The positive side of social comparison on social network sites: How envy can drive inspiration on Instagram, Cyberpsychol. Behav. Soc. Netw. 21 (2018) 411417. https://doi.org/10.1089/cyber.2017.0708. 
[31] E.J. Noon, A. Meier, Inspired by friends: Adolescents' network homophily moderates the relationship between social comparison, envy, and inspiration on Instagram, Cyberpsychol. Behav. Soc. Netw. 22 (2019) 787-793. https://doi.org/10.1089/cyber.2019.0412.

[32] S. Ruggieri, S. Ingoglia, R.C. Bonfanti, G. Lo Coco, 2021. The role of online social comparison as a protective factor for psychological wellbeing: A longitudinal study during the COVID-19 quarantine. Personality and Individual Differences 171, 110486. https://doi.org/10.1016/j.paid.2020.110486.

[33] R. Lin, S. Utz, The emotional responses of browsing Facebook: Happiness, envy, and the role of tie strength, Computers in Human Behavior 52 (2015) 29-38. https://doi.org/10.1016/j.chb.2015.04.064.

[34] I. Beyens, J.L. Pouwels, I.I. van Driel, L. Keijsers, P.M. Valkenburg, The effect of social media on well-being differs from adolescent to adolescent, Sci Rep 10 (2020) 1-11. https://doi.org/10.1038/s41598-020-67727-7.

[35] I. Beyens, J.L. Pouwels, I.I. van Driel, L. Keijsers, P.M. Valkenburg, Social media use and adolescents' well-being: Developing a typology of person-specific effect patterns, Communication Research (2021). https://doi.org/10.1177/00936502211038196.

[36] D. Schmuck, K. Karsay, J. Matthes, A. Stevic, “Looking up and feeling down”. The influence of mobile social networking site use on upward social comparison, self-esteem, and well-being of adult smartphone users, Telematics and Informatics (2019). https://doi.org/10.1016/j.tele.2019.101240.

[37] D. Wirtz, A. Tucker, C. Briggs, A.M. Schoemann, How and why social media affect subjective well-being: Multi-site use and social comparison as predictors of change across time, J Happiness Stud 22 (2021) 1673-1691. https://doi.org/10.1007/s10902-020-00291-z. 
[38] M.T. Kohler, I.N. Turner, G.D. Webster, Social comparison and state-trait dynamics: Viewing image-conscious Instagram accounts affects college students' mood and anxiety, Psychology of Popular Media 10 (2021) 340-349. https://doi.org/10.1037/ppm0000310.

[39] G. Valerio-Ureña, A.L.M. Gómez, G.V. Flores, Dispositional envy, satisfaction with life and images shared on Instagram by university students, in: Proceedings of the $20208^{\text {th }}$ International Conference on Information and Education Technology, Okayama Japan, ACM, New York, NY, USA, 2020, pp. 196-199.

[40] J. Nesi, A.B. Miller, M.J. Prinstein, Adolescents' depressive symptoms and subsequent technology-based interpersonal behaviors: A multi-wave study, J. Appl. Dev. Psychol. 51 (2017) 12-19. https://doi.org/10.1016/j.appdev.2017.02.002.

[41] J. Chae, Explaining females' envy toward social media influencers, Media Psychology 21 (2018) 246-262. https://doi.org/10.1080/15213269.2017.1328312.

[42] G. Aalbers, R.J. McNally, A. Heeren, S. de Wit, E.I. Fried, Social media and depression symptoms: A network perspective, J. Exp. Psychol. Gen. 148 (2019) 1454-1462. https://doi.org/10.1037/xge0000528.

[43] S. Scherr, C.L. Toma, B. Schuster, Depression as a predictor of Facebook surveillance and envy, Journal of Media Psychology 31 (2018) 196-202. https://doi.org/10.1027/1864-

\section{$\underline{1105 / a 000247 .}$}

*[44] E.C. Tandoc, Z.H. Goh, Is Facebooking really depressing? Revisiting the relationships among social media use, envy, and depression, Information, Communication \& Society (2021). https://doi.org/10.1080/1369118X.2021.1954975.

This three-wave panel survey found evidence for a reinforcing envy-depression spiral on SM.

Results show that time spent on Facebook increased depressive symptoms a year later, which 
increased time on Facebook another year later. Time on Facebook also predicted later envy, which in turn predicted later depressive symptoms.

[45] L. Faelens, K. Hoorelbeke, B. Soenens, K. van Gaeveren, L. de Marez, R. de Raedt, E.H. Koster, Social media use and well-being: A prospective experience-sampling study, Computers in Human Behavior 114 (2021) 106510. https://doi.org/10.1016/j.chb.2020.106510.

[46] P.S. Dodds, E.M. Clark, S. Desu, M.R. Frank, A.J. Reagan, J.R. Williams, L. Mitchell, K.D. Harris, I.M. Kloumann, J.P. Bagrow, K. Megerdoomian, M.T. McMahon, B.F. Tivnan, C.M. Danforth, Human language reveals a universal positivity bias, Proc. Natl. Acad. Sci. U. S. A. 112 (2015) 2389-2394. https://doi.org/10.1073/pnas.1411678112.

[47] M. Faranda, L.D. Roberts, Social comparisons on Facebook and offline: The relationship to depressive symptoms, Personality and Individual Differences 141 (2019) 13-17. https://doi.org/10.1016/j.paid.2018.12.012.

[48] E. Wallace, I. Buil, 2021. Hiding Instagram likes: Effects on negative affect and loneliness. Personality and Individual Differences 170, 110509. https://doi.org/10.1016/j.paid.2020.110509.

**[49] J. Kang, B. Liu, A similarity mindset matters on social media: Using algorithm-generated similarity metrics to foster assimilation in upward social comparison, Social Media + Society 5 (2019) 1-15. https://doi.org/10.1177/2056305119890884.

This experiment tests whether showing Facebook users a (supposedly) algorithmically generated similarity metric next to a user profile changes the effects of upward comparison to a physically attractive or financially successful target. Results suggest that moderate similarity cues may improve both target liking and user well-being. 
[50] B.M. Trifiro, J. Gerson, Social media usage patterns: Research note regarding the lack of universal validated measures for active and passive use, Social Media + Society 5 (2019) 14. https://doi.org/10.1177/2056305119848743.

[51] S. Choi, E.-M. Kim, Between Instagram browsing and subjective well-being: Social comparison or emotional contagion?, Media Psychology 24 (2021) 866-890. https://doi.org/10.1080/15213269.2020.1824120.

*[52] B.K. Johnson, S. Knobloch-Westerwick, When misery avoids company: Selective social comparisons to photographic online profiles, Hum Commun Res 43 (2017) 54-75. https://doi.org/10.1111/hcre.12095.

Two selective exposure experiments demonstrate how users differentially gravitate towards photographic online profiles depending on prior mood. Findings underline that users do make selective, self-enhancing (albeit not necessarily conscious) choices about which content they view on SM, with implications for their well-being.

[53] J.W. Ouwerkerk, B.K. Johnson, Motives for online friending and following: The dark side of social network site connections, Social Media + Society 2 (2016) 1-13. https://doi.org/10.1177/2056305116664219.

[54] E.K. Yuen, E.A. Koterba, M.J. Stasio, R.B. Patrick, C. Gangi, P. Ash, K. Barakat, V. Greene, W. Hamilton, B. Mansour, The effects of Facebook on mood in emerging adults, Psychology of Popular Media Culture 8 (2019) 198-206. https://doi.org/10.1037/ppm0000178.

[55] B.K. Johnson, Look up, look down: Articulating inputs and outputs of social media social comparison, JoCTEC 4 (2021). https://doi.org/10.51548/joctec-2021-003. 
[56] B. Naderer, C. Peter, K. Karsay, This picture does not portray reality: Developing and testing a disclaimer for digitally enhanced pictures on social media appropriate for Austrian tweens and teens, Journal of Children and Media (2021).

https://doi.org/10.1080/17482798.2021.1938619.

[57] S. Weber, T. Messingschlager, J.-P. Stein, This is an Insta-vention! Exploring cognitive countermeasures to reduce negative consequences of social comparisons on Instagram, Media Psychology (2021). https://doi.org/10.1080/15213269.2021.1968440. 